\title{
Inquérito epidemiológico de tracoma em escolares no município de Embu das Artes - SP
}

\author{
Trachoma epidemiological school survey in the city of Embu das Artes - SP
}

Paulo Antonio Caninéo ${ }^{1}$, Shizuko Nishimura², Norma Helen Medina ${ }^{3}$, Inês Kazue Koizumi ${ }^{4}$, Maria Regina Alves Cardoso 5

\section{RESUMO}

Objetivo: Estimar a prevalência do tracoma em escolares no município de Embu das Artes - SP, visando a implementação das ações de vigilância epidemiológica da doença.

Métodos: O município de Embu das Artes - SP dista 25 km da capital. Nos anos 20032004 foi realizado um inquérito por amostragem por conglomerados de escolares com a mesmo método do inquérito nacional de tracoma em escolares do Ministério da Saúde. Previamente aos trabalhos de busca ativa, foram realizadas atividades de educação em saúde em todas as escolas. Foi realizado exame ocular externo em todos os alunos para a detecção do tracoma, segundo os critérios da OMS. Todos os casos de tracoma foram tratados, notificados e seus familiares investigados.

Resultados: Foram examinados 2.374 alunos de nove escolas públicas sorteadas. A prevalência de tracoma inflamatório folicular (TF) foi 3,1\% (IC 95\%: 2,4\% - 3,9\%), variando de $0,5 \%$ a $4,2 \%$ nas escolas examinadas. Foi encontrada maior prevalência 8,6\% em crianças com 6 anos de idade.

Conclusão: A doença comportou-se de forma branda neste município, pois não foram detectados casos de tracoma intenso nem tracoma cicatricial. Todavia, a prevalência foi maior que a encontrada no município de São Paulo. As ações de vigilância epidemiológica do tracoma devem ser contínuas, principalmente nos locais onde foram encontradas as maiores prevalências.

Descritores: Tracoma/epidemiologia; Tracoma/prevenção \& controle; Vigilância epidemiológica; Saúde escolar; Chlamydia trachomatis

\section{ABSTRACT}

Purpose: To estimate the trachoma prevalence in school children in Embu das Artes - SP, aiming the implementation of the disease epidemiological surveillance.

Methods: The city of Embu das Artes - SP, is $25 \mathrm{~km}$ far from the capital of the State. In the years of 2003-2004, a trachoma survey was conducted in a cluster sample of school children with the same methodology of the national trachoma student's survey of the Ministry of Health. Previously to the trachoma active search, activities of healtheducation were performed in all schools. External ocular examinations were done in all students to detect trachoma according to the WHO criteria. All cases of trachoma were notified and their families were submitted to an external ocular examination. The cases were treated with $1 \%$ tetracycline ointment or systemic azithromycin.

Results: 2,374 students from nine sampled selected public schools were examined. The prevalence of follicular inflammatory trachoma (TF) was 3.1\% (IC 95\%: 2.4-3.9), varying from $0.5 \%$ to $4.2 \%$ in the examined schools. The prevalence for males was $3.2 \%$ and for females was 3.0\%. The greater prevalence (8.6\%) was found in 6 year-old children.

Conclusion: The disease showed a mild behavior in this city, because no cases of intense inflammatory trachoma or cicatricial trachoma were detected. However, the prevalence was greater than the one found in the city of São Paulo. Epidemiological surveillance activities of trachoma must be continuous, mainly in places where the greater prevalence had been found.

Keywords: Trachoma/epidemiology; Trachoma/prevention \& control; Epidemiological surveillance; School health; Chlamydia trachomatis

\section{INTRODUÇÃO}

O tracoma é uma inflamação crônica da conjuntiva e da córnea causada pela Chlamydia trachomatis. A inflamação ativa afeta, principalmente, pré-escolares e escolares, e tem início na forma de conjuntivite folicular, com hipertrofia papilar e infiltração inflamatória na conjuntiva tarsal superior ${ }^{(1)}$.

A doença pode evoluir com o aparecimento de cicatrizes na conjuntiva e nos casos mais graves as cicatrizes se confluem, provocando deformidades nas pálpebras, causando entrópio e triquíase. Essas lesões produzem abrasões corneanas por ação mecânica, podendo levar à perda da visão(1).
O tracoma é descrito em locais com precárias condições de higiene, grande concentração populacional, saneamento básico deficiente e baixos níveis educacional e populacional(2)

As atividades de vigilância epidemiológica são direcionadas para os grupos de maior risco de desenvolver a doença que são as crianças até 10 anos de idade ${ }^{(3)}$.

No Brasil a doença ocorre de forma endêmica em várias regiões, principalmente em áreas rurais e comunidades indígenas ${ }^{(4,5)}$. É uma doença de notificação compulsória no Estado de São Paulo, tem seu registro no Sistema Nacional de Agravos de Notificação (Sinan Net) e é um indicador pactuado pelos estados e municípios na Programação

Médico, Programa de Tracoma e Hanseníase Ocular, Secretaria Municipal de Embu das Artes Embu das Artes (SP), Brasil.

Médica, Grupo de Vigilância Epidemiológica de Osasco (GVE - X), Centro de Vigilância Epidemiológica "Prof. Alexandre Vranjac", Coordenadoria de Controle de Doenças, Secretaria de Estado da Saúde de São Paulo - São Paulo (SP), Brasil.

Médica, Centro de Oftalmologia Sanitária, Centro de Vigilância Epidemiológica "Prof. Alexandre Vranjac", Coordenadoria de Controle de Doenças, Secretaria de Estado da Saúde São Paulo - São Paulo (SP), Brasil.

${ }^{4}$ Médica Sanitarista, Divisão de Métodos e Capacitação em Epidemiologia, Centro de Vigilância Epidemiológica "Prof. Alexandre Vranjac", Coordenadoria de Controle de Doenças - Secretaria de Estado da Saúde São Paulo - São Paulo (SP), Brasil.

Médica, Departamento de Epidemiologia, Faculdade de Saúde Pública, Universidade de São Paulo - USP - São Paulo (SP), Brasil.

Financiamento: Não houve financiamento para este trabalho.

Divulgação de potenciais conflitos de interesse: P.A.Caninéo, Nenhum, S.Nishimura, Nenhum N.H.Medina, Palestrante pela Fundação Paulista de Hanseníase, I.K.Koizumi, Nenhum, M.R.A.Cardoso, Nenhum.

Endereço para correspondência: Paulo Antonio Caninéo, Rua Caraibas, 400/112, São Paulo (SP) -05020-000 - Brasil - E-mail: paulo.antonio31@terra.com.br 
de Ações de Vigilância em Saúde (PAVS), para a liberação de financiamento pelo Ministério da Saúde ${ }^{(6,7)}$.

A prevalência do tracoma é menor no Estado de São Paulo do que nos estados do Norte e Nordeste, porém alguns municípios de São Paulo apresentam prevalências maiores de 10\% em escolares ${ }^{(8-10)}$.

A Organização Mundial da Saúde (OMS) lançou um programa de eliminação do tracoma como causa de cegueira até o ano de 2020(11). Para a certificação da eliminação é necessário que se identifiquem todos os locais, onde a prevalência é alta, para intensificar as atividades de tratamento e controle com a meta de diminuir a prevalência de tracoma ativo em crianças menores de 10 anos para menos de $5 \%{ }^{(12)}$.

Com o objetivo de estimar a prevalência do tracoma em escolares no município de Embu das Artes, SP visando à implementação das ações de vigilância epidemiológica da doença, foi realizado um inquérito epidemiológico em uma amostra de alunos de 1a a 4a série do ensino fundamental de escolas públicas.

\section{MÉTODOS}

O município de Embu das Artes - SP, situa-se na região sudoeste da Região Metropolitana de São Paulo, dista $25 \mathrm{~km}$ da capital e faz parte do Grupo de Vigilância Epidemiológica Regional Osasco (GVE - X) que compreende 15 municípios (Figura 1). Sua população segundo dados do IBGE, era de 206.781 habitantes no ano de 2000 (13).

Foi selecionada uma amostra probabilística da população de alunos da 1a a $4^{a}$ série do ensino fundamental de escolas da rede pública. Utilizou-se o censo do Ministério da Educação (MEC) da população de escolares de 1a a 4a série da rede oficial de ensino público do ano anterior ao inquérito ${ }^{(14)}$.

O tamanho da amostra foi estimado considerando-se uma prevalência de 5\% de tracoma em todas as suas formas clínicas, aceitandose um erro máximo de amostragem de 0,01 em 95\% das possíveis amostras. Foram acrescidos 20\% no tamanho para a correção de perdas e faltosos. O desenho amostral foi aleatório por conglomerados, sendo o período de estudo a unidade amostral ${ }^{(4)}$.

Previamente aos trabalhos de busca ativa, foram realizadas atividades de educação em saúde em todas as escolas, inclusive com distribuição de folhetos educativos.

O exame ocular externo foi realizado por examinadores padronizados que receberam treinamento prévio para realização de todos os procedimentos da pesquisa. Foi usada lupa de pala 2,5X de aumento com iluminação natural ou artificial com lanterna para detectar a presença de sinais clínicos de tracoma, segundo o Esquema Simplificado de Gradação da OMS ${ }^{(15)}$.

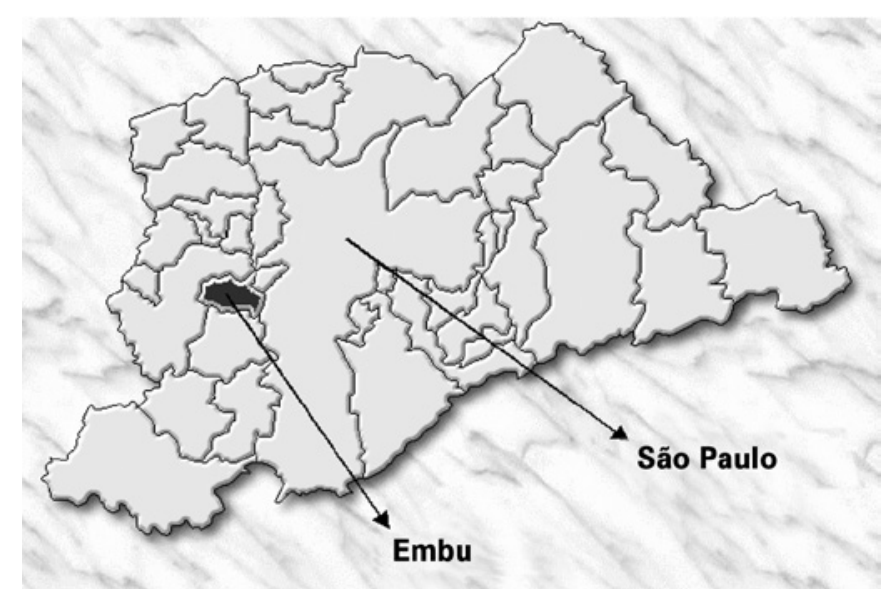

Figura 1. Mapa da localização do município em relação à Região Metropolitana de São Paulo.
Foi considerado caso de tracoma a criança que apresentasse uma ou mais das seguintes formas clínicas: TF - tracoma inflamatório folicular, TI - tracoma inflamatório intenso, TS - tracoma cicatricial, TT - triquíase tracomatosa, CO - opacificação de córnea(15).

Os dados foram coletados nas planilhas de inquérito/busca ativa do Ministério da Saúde onde foram registrados dados sobre a escola e sobre os alunos da amostra, como: nome, sexo, idade, presença e forma clínica de tracoma e conjuntivites associadas ${ }^{(4,16)}$.

Os comunicantes domiciliares dos casos positivos foram submetidos a exame ocular externo para detecção de outros casos relacionados.

Todos os casos de tracoma foram notificados ao sistema de vigilância epidemiológica por meio de Fichas de Investigação Epidemiológica, instituído o tratamento e orientação sobre a doença, sua prevenção, tratamento e controle ${ }^{(17)}$.

O tratamento preconizado foi a aplicação de tetraciclina 1\%, pomada oftálmica, duas vezes ao dia, por 45 dias consecutivos ou azitromicina $20 \mathrm{mg} / \mathrm{kg}$ de peso em dose única, via oral, fornecida pela Secretaria de Estado da Saúde de São Paulo. Todos os casos foram acompanhados para controle de cura por um ano(3,16).

Foram reforçadas as seguintes medidas de prevenção: separar e manter a higiene de objetos de uso pessoal, usar lenços e toalhas de papel descartável, lavar as mãos e o rosto, como também cuidados para diminuir a população de moscas e mosquitos ${ }^{(3,11,17)}$.

Os dados coletados nas planilhas foram supervisionados antes de serem digitados foram analisados usando o aplicativo EPI-INFO.

Este inquérito de tracoma foi aprovado pelo Comitê de Ética do Instituto Adolpho Lutz, da Secretaria de Estado da Saúde, São Paulo.

\section{RESULTADOS}

O trabalho de campo foi realizado nos anos de 2003 e 2004. A amostra total de alunos foi de 2.952 , sendo examinados 2.374 alunos de 1a a 4a série das nove escolas públicas sorteadas do município, apresentando uma perda da amostra de 19,6\%

Foram diagnosticados 73 casos de tracoma inflamatório folicular (TF), resultando na prevalência de 3,1\% (IC 95\%: 2,4\% - 3,9\%), variando de $0,5 \%$ a $4,2 \%$ nas escolas examinadas. Não foram diagnosticados casos de outras formas clínicas da doença. A prevalência de TF para o sexo masculino foi 3,2\% e para o feminino 3,0\%, diferença não significante $(p=0,39)$.

Foi encontrada maior prevalência de tracoma $(8,6 \%)$ em criancas com 6 anos de idade, diminuindo conforme o aumento da idade (Gráfico). A prevalência de TF em alunos com 10 anos ou menos foi de 3,4\%.

Foram examinados 127 comunicantes domiciliares sendo diagnosticados $9(7,0 \%)$ casos de tracoma.

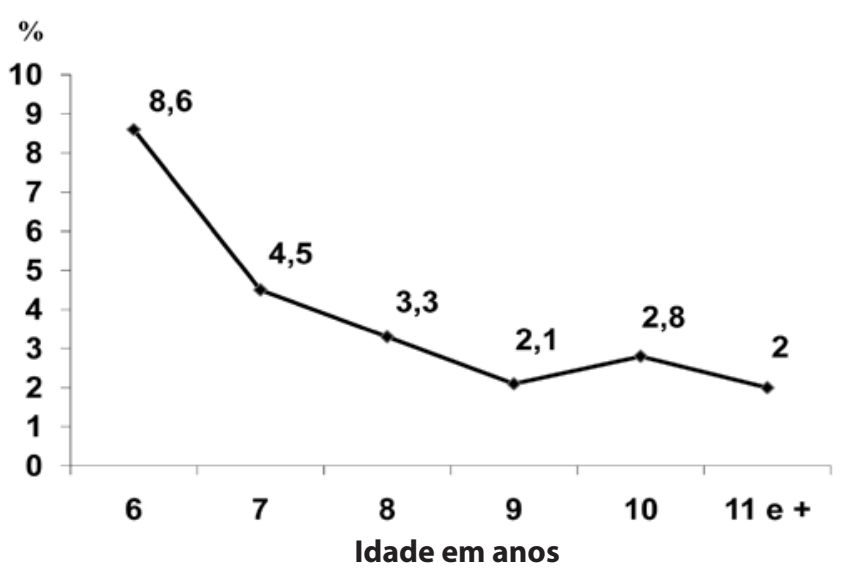

Gráfico. Prevalência de tracoma inflamatório folicular (TF), por idade, em amostra de escolares. Embu das Artes - SP, 2003-2004. 


\section{DISCUSSÃO}

Este estudo foi realizado em uma amostra de escolares, que é a maneira mais fácil de localizar as crianças nessa faixa etária em uma comunidade. Em vista desse método, não é possível estimar a prevalência entre crianças que não frequentam as escolas e nem entre as crianças de 1 a 5 anos de idade. A perda da amostra foi devido aos faltosos, apesar das várias visitas realizadas nas escolas.

A prevalência de tracoma encontrada foi maior que aquela observada no município de São Paulo em 1999 (2,2\%), mas foi menor que a do Estado de São Paulo em 2002 (4,1\%) e de outros municípios do interior, como Cajobi (9,6\%), Guaraci (6,9\%) e Olímpia $(5,5 \%)$. Este resultado foi semelhante ao inquérito escolar por amostragem realizado em Botucatu, SP $(2,9 \%)^{(8,18-20)}$. Quando estes dados são comparados ao de outros locais do Brasil, a prevalência é mais baixa que Duque de Caxias, RJ (8,2\%), Joinville, SC $(4,9 \%)$ e Manaus, AM (4,8\%)(21-23).

A doença comportou-se de forma branda no município de Embú das Artes, pois não foram detectados casos de tracoma inflamatório intenso nem de tracoma cicatricial. Porém, o coeficiente de detecção nos comunicantes domiciliares examinados foi alto, concordando com os achados no município de São Paulo, indicando que existe transmissão intradomiciliar ${ }^{(18)}$.

A prevalência de tracoma ativo maior na faixa etária de 6 anos está de acordo com a literatura, a qual mostra que o tracoma ativo é mais prevalente em pré-escolares ${ }^{(2)}$. E mostra que se deve intensificar os trabalhos educativos e de vigilância epidemiológica priorizando essa faixa etária, como também demonstra que há necessidade de realizar busca ativa em crianças de 1 a 5 anos nos domicílios, creches e pré-escolas.

\section{CONCLUSÃO}

A presença de casos de tracoma no município de Embu das Artes, com prevalência superior a do município de São Paulo, e a possibilidade de disseminação da doença, principalmente em pré-escolares e escolares, evidencia a importância da continuidade das ações de prevenção e de controle desta doença. As ações de vigilância epidemiológica do tracoma devem ser priorizadas nas escolas, onde foram encontradas as maiores prevalências e nos domicílios dos casos de tracoma diagnosticados.

\section{AGRADECIMENTOS}

Aos profissionais de saúde que colaboraram com a investigação, principalmente o trabalho incansável da Sra. Dalila Magdalena Silva de Moraes in memorium e da Sra. Helena Gomes Stadler.

\section{REFERÊNCIAS}

1. Dawson CR, Jones BR, Tarizzo ML. Guía Práctica de lucha contra el tracoma. Genebra: Organização Mundial de Saúde; 1981.

2. World Health Organization - WHO. Primary health care level management of trachoma. Geneve; 1989. 14p

3. Brasil. Ministério da Saúde.Fundação Nacional de Saúde. Manual de Controle do Tracoma, Brasília (DF) 2001. 56p.

4. Lopes MF, Medina NH, Luna JE, Freitas HAS, Koizumi IK, Brock KR, et al. Tracoma: situação epidemiológica no Brasil. Arq Bras Oftalmol. 2008;71(4 Supl):7-19.

5. Freitas HS, Lopes MFC, Medina NH et al. Ocorrência de tracoma em comunidades indígenas brasileiras. Arq Bras Oftalmol, 2009;72(4 Supl):25.

6. São Paulo (Estado). Resolução S.S. no 60/92 de 17 de fev. de 1992, alterada D.O.E. 104 (162). Dispõe sobre a lista de doenças de notificação compulsória. Diário Oficial do Estado de São Paulo, 1994; São Paulo. 21p.

7. Brasil. Ministério da Saúde. Secretaria de Vigilância em Saúde. Portaria no 64, de 30 de maio de 2008. Estabelece a programação das ações de vigilância em Saúde (PAVS). [citado 2011 Ago 23]. Disponível em: http://bvsms.saude.gov.br/bvs/saudelegis/ svs/2008/prt0064_30_05_2008.html

8. Medina NH. Tracoma no Estado de São Paulo. Bol Epidemiol Paul. (BEPA) 2004; 1 n(7):12-13.

9. Scarpi MJ, Plutt RC, Arruda HO. Prevalência de tracoma no povoado de Mocambo, Estado do Ceará, Brasil. Arq Bras Oftalmol. 1989;52(5):177-9.

10. Garrido C, Guidugli T, Campos M. Estudo clínico-laboratorial do tracoma em população indígena da Amazônia brasileira. Arq Bras Oftalmol. 1999;62(2):132-8.

11. Organización Mundial de la Salud. Programa de Prevención de la Ceguera y de la Sordera. Informe de una Reunión Científica Mundial "Lucha Contra el Tracoma: Perspectivas. WHO/PBL/96.56. Geneva: OMS; 1997.

12. World Health Organization. Report of the $2^{\text {nd }}$ Global Scientific Meeting on Trachoma. WHO/PBD/GET.03.1. Geneva: WHO; 2003.

13. Brasil. Instituto Brasileiro de Geografia e Estatística [Internet]. [citado 2012 Ago 3].Disponível em: www.ibge.gov.br

14. Brasil. Ministério da Educação. Censo Escolar de 1aa a 4⿳亠丷厂 series do ensino fundamental. Brasília (DF); 2003.

15. Thylefors B, Dawson CR, Jones BR, West SK, Taylor HR. A simple system for assessment of trachoma and its complications. Bull World Health Organ. 1987;65(4):477-83.

16. Brasil. Ministério da Saúde. Inquérito epidemiológico de tracoma em escolares - manual de procedimentos [Internet]. [citado 2012 Ago 3]. Brasília(DF); 2002. Disponível em: ftp://ftp.cve.saude.sp.gov.br/doc_tec/outros/tracoma_inque.pdf.

17. São Paulo. Secretaria de Estado da Saúde de São Paulo. Manual de vigilância epidemiológica do tracoma: normas e instruções. 2aed. São Paulo;1993.

18. Koizumi IK, Medina NH, D'Amaral RK, Morimoto WT, Caligaris LS, Chinen N, et al. Prevalência de tracoma em pré-escolares e escolares no Município de São Paulo. Rev Saúde Pública. 2005;39(6):937-42.

19. Medina NH, Oliveira MB, Tobin S, Kiil G Jr, Mendoca MM, de Barros OM, Newland HS, et al.. The prevalence of trachoma in preschool and school children in Olímpia, Guaraci and Cajobi, São Paulo, Brazil. Trop Med Parasitol. 1992;43(2):121-3.

20. Schellini SA, Lavezzo MM, Ferraz LB, Neto JO, Medina NH, Padovani CR. Prevalência e localização espacial dos casos de tracoma detectados em escolares de Botucatu, São Paulo - Brasil. Arq Bras Oftalmol. 2010;73(4):358-62.

21. Couto Junior AS, Scarpi MJ, Guidugli, T. Prevalência de tracoma em escolares e préescolares no Município de Duque de Caxias, RJ. Rev Bras Oftalmol. 1997;56(7):515-21.

22. Nobrega MJ, Farah AL, Miller D, Kang HM, Haddad LH. Estudo clínico e laboratorial de tracoma em escolares de Joinville, Santa Catarina, Brasil. Arq Bras Oftalmol. 1998; 61(5):551-6.

23. Carvalho RC, Falcão R, Cohen J,Chaves C, Scarpi MJ. Prevalência de tracoma em escolares de Manaus, Amazonas, Brasil. Arq Bras Oftalmol. 1997;60(3):243-7. 